\title{
Retrospective study of MRI images to examine the effects of estrogen supplementation on breast tissue: A pilot study in Asian Taiwan
}

\author{
Ting-Kai Leung ${ }^{1,2,3^{*}}$, Pai-Jung Huang ${ }^{2,3}$, Chih-Hsiung $\mathrm{Wu}^{2,3}$, Chi-Ming Lee ${ }^{1,2,3}$, \\ Chin-Sheng Hung ${ }^{3}$, Hung-Hua Liang ${ }^{3}$, Jeng-Fong $\mathrm{Chiou}^{3}$ \\ ${ }^{1}$ Department of Diagnostic Radiology, Taipei Medical University Hospital, Taipei City, Chinese Taipei; \\ *Corresponding Author: hk8648@tmu.edu.tw \\ ${ }^{2}$ Breast Health Center, Taipei Medical University Hospital, Taipei City, Chinese Taipei \\ ${ }^{3}$ Faculty of Medicine, Taipei Medical University, Taipei City, Chinese Taipei
}

Received 6 May 2013; revised 7 June 2013; accepted 12 July 2013

Copyright (c) 2013 Ting-Kai Leung et al. This is an open access article distributed under the Creative Commons Attribution License, which permits unrestricted use, distribution, and reproduction in any medium, provided the original work is properly cited.

\begin{abstract}
MRI provides a highly sensitive technique for early detection of abnormal breast tissue. It is useful for identifying a status of proliferation, angiogenesis, and microvascular permeability, which may indicate early breast neoplasm formation. We retrospectively studied 2005 breast MRI images from Taiwanese women and classified them as either healthy or unhealthy according to BI-RADS categories. A subgroup of our study patients had received estrogen supplements, containing estrogen components or phytoestrogen, for at least 3 months. These patients' images were also classified into the healthy and unhealthy groups. These two groups were compared and a significant difference was found between them $(P<0.002)$. Comparison of the MRI images also identified certain cases that demonstrated a typical estrogen/phytoestrogen effect or a withdrawal effect. The overuse of estrogen or phytoestrogen supplements can increase breast glandular tissue proliferation, as reflected on MRI images. Such proliferation may increase the patient's risk of future breast cancer.
\end{abstract}

Keywords: Breast MRI; Estrogen; Hormone Effect; Phytoestrogen; Proliferative Breast Glandular Tissue

\section{INTRODUCTION}

In certain Western countries, such as Germany, hormone replacement therapy (HRT) is widely recognized as a risk factor for breast cancer. Since 2000 the use of
HRT has decreased substantially in the general population in Germany, and a corresponding decline was noted in breast cancer incidence between 2000 and 2005 [1]. This finding clearly reflects the positive relationship between HRT and incidence of breast cancer.

By contrast, the incidence of breast cancer has risen sharply in the past 2 decades, with a corresponding increase in mortality from breast cancer in Taiwan. The increase during this period has been twofold or threefold and shows no signs of slowing. Possible reasons for this increase include lifestyle changes and greater westernization.

Taiwanese breast cancer patients tend to be younger than their European or American counterparts [2]. In Taiwan, approximately $50 \%$ of clinically diagnosed cases of breast cancer occur in patients aged under 50 years. By contrast, the majority of newly diagnosed breast cancer patients in Western countries are older than 50 years. In addition, the age-specific incidence of breast cancer in Taiwan rapidly increases in patients aged approximately 45 years. The equivalent peak in diagnosis occurs 5 to 10 years later among patients in the United States and Europe [2]. The higher incidence of breast cancer in premenopausal women in Taiwan may reflect the relationship between breast cancer and estrogen supplements, which may have unique patterns of use among in Taiwanese women.

Magnetic resonance imaging (MRI) of the breast is extremely sensitive for detecting lesions. It can identify the abnormal statuses of proliferation, angiogenesis, and microvascular permeability that occur in early breast neoplasm formation. In the current study we retrospectively reviewed breast MRI images for patients with a known estrogen and phytoestrogen intake to investigate a 
possible associated cancer risk. We assessed the effect of estrogen and phytoestrogen on breast glandular tissue, as shown by MRI results.

\section{MATERIAL AND METHODS}

\subsection{Total Study Population and Comparison Group}

We collected retrospective data from 2080 MRI images of women who attended the Breast Health Center at Taipei Medical University, Taipei, between December 2008 and December 2011. Women who received MRI around the time of menstruation (3 days before, up to 7 days after) were excluded to reduce the possibility of endogenous estrogen affecting the image results.

\subsection{Experimental Group}

The experimental group formed a subgroup within the total study group. A total of 121 images of women were selected for the experimental group and had attended the clinic between September 2010 and December 2011. These women reported having received estrogen supplements containing mimic estrogen components or phytoestrogen (including herbs or Chinese herbs such as genistein, royal jelly, evening primrose oil, yam extract, and Ganoderma lucidum) for at least 3 months prior to their MRI.

\subsection{Image Acquisition}

Pre-enhanced and post-enhanced subtracted images were obtained, and an enhanced curve analysis was performed. All imaging studies were performed on a $1.5 \mathrm{~T}$ dedicated spiral breast MRI system (Aurora Systems, USA). For the screening procedure, patients were placed in a prone position, feet first, on the table inside the DBMRI machine. Breasts were fitted and positioned using a breast transmission-receiving (TR) coil. The offresonance spiral image mode was used. A gradient echo with $10 \mathrm{~ms}$ of TR and T2-weighted spin echo with 2.5 ms of TR were acquired. The echo time was $5 \mathrm{~ms}$ for RODEO and $120 \mathrm{~ms}$ for the T2 sequences. The matrix size was $512 \times 512$. The slice thickness was $1.1 \mathrm{~mm}$. The field of view of each station was $20 \mathrm{~cm}$ to $36 \mathrm{~cm}$. Sequences were performed before and after the infusion of $0.2 \mathrm{mmol} / \mathrm{kg}$ Magnevist (Bayer-Schering Pharma AG, Germany), administered as a bolus dose with a power injector, followed by a $20-\mathrm{mL}$ saline flush. Post-processing of data was displayed by AuroraCad 4.0 (Aurora System, North Andover, MA, USA).

\subsection{BIRADS Grading of Breast MRI Interpretations}

The breast image reporting and data system (BI-RADS) was used, and the interpretation of BRMRI also adopts the standards of the ACR BIRADS-MRI lexicon [3-5]. Image results scored as either a BI-RADS category 1 or 2 imply an interpretation of normal and benign findings, with no risk of malignancy. If the image result is scored as category 3 (probably benign), category 4 (suspicious abnormality), category 5 (highly suggestive of malignnancy), or category 6 (already known malignancy), these interpretations indicate the patient is at risk for malignnancy.

\subsection{Classification of Healthy vs. Unhealthy Breast Interpretations}

We used a simple classification system in which the "healthy” images received an MRI rating of BI-RADS categories I and II, and "unhealthy" images were rated as BI-RADSIII, IV, V, or VI.

\subsection{Statistical Comparison of Healthy and Unhealthy Breast Images}

All analyses were performed with SPSS statistical software. We used a one-sample binomial test to compare the outcomes of long-term (at least 3 months) estrogen and phytoestrogen use (experimental group) versus the comparison group (study population). A $P$-value of less than 0.05 was considered statistically significant.

\section{RESULTS}

\section{Ratio of Healthy to Unhealthy Images}

We analyzed the results for all 2080 images of women who had received breast MRI screening at our hospital. We classified $50.86 \%$ of the images as healthy; thus, unhealthy images accounted for $49.14 \%$ of our study population (Table 1) [2,6-8]. Within the total study group, a subgroup of 121 images of women had reported taking estrogen or phytoestrogen regularly for at least 3 months. We retrospectively reviewed their MRI results and classified $38.01 \%$ of the breast images from this subgroup as healthy. Thus, for the subgroup who reported having taken estrogen supplements, 61.99\% of the breast images were classified unhealthy (Table 1).

We used SPSS to perform a single-sample binomial test to compare the results of the estrogen users versus the overall study population. The results showed a statistically significant difference $(P<0.002)$, indicating that women taking estrogen or phytoestrogen supplements had a greater tendency to develop breast disease or unhealthy breast conditions.

Based on our experience of assessing over 2000 breast-screening MRI images, we found that an intake of external estrogen is one factor that may enhance proliferative changes in breast glandular tissue. In the current 
study, cases among the subgroup (experimental group) included women who were using synthetic estrogen for HRT and others who used herbal supplements or Chinese herbs containing estrogen components or phytoestrogen. Our results indicated that natural supplements such as genistein, royal jelly, evening primrose oil, yam extract, and Ganoderma lucidum could stimulate breast glandular tissue in the same way as HRT, yielding a positive proliferative change, detectable on MRI images.

Figure 1 illustrates selected cases from our observations. In these images, the effects of estrogen or phytoestrogen intake are visible, as is the effect of ovariectomy. Both these factors have been found to significantly affect the results of MRI imaging, with estrogen intake being associated with increased breast glandular tissue postGD enhancement. Cases 2 and 3 showing breast MRI scans taken before and after the patient's intake of estrogen or phytoestrogen for comparison of interval MRI pictures, note the newly enhanced foci and tumor after estrogen intake.

By contrast, Figure 2 illustrates the decreased proliferation and the normalization of breast glandular tissues

Table 1. Comparison of MRI images in the study population and a subgroup of estrogen users.

\begin{tabular}{cccc}
\hline & $\begin{array}{c}\text { Healthy breast } \\
\text { MRI results } \\
\text { (BIRADS I , II ) }\end{array}$ & $\begin{array}{c}\text { Unhealthy breast } \\
\text { MRI results } \\
\text { (BIRAD 0, III, IV, }\end{array}$ & $P$ \\
\hline $\begin{array}{c}\text { All women screened } \\
(\mathrm{n}=2080)\end{array}$ & $1058(50.86 \%)$ & $1022(49.14 \%)$ & \\
$\begin{array}{c}\text { Women taking estrogen } \\
\text { or phytoestrogen for at }\end{array}$ & $46(38.01 \%)$ & $75(61.99 \%)$ & $\mathbf{0 . 0 0 2}^{* *}$ \\
least 3 months (n=121) & & & \\
\hline
\end{tabular}

${ }^{* *}$ Statistically significant at $P<0.01$.
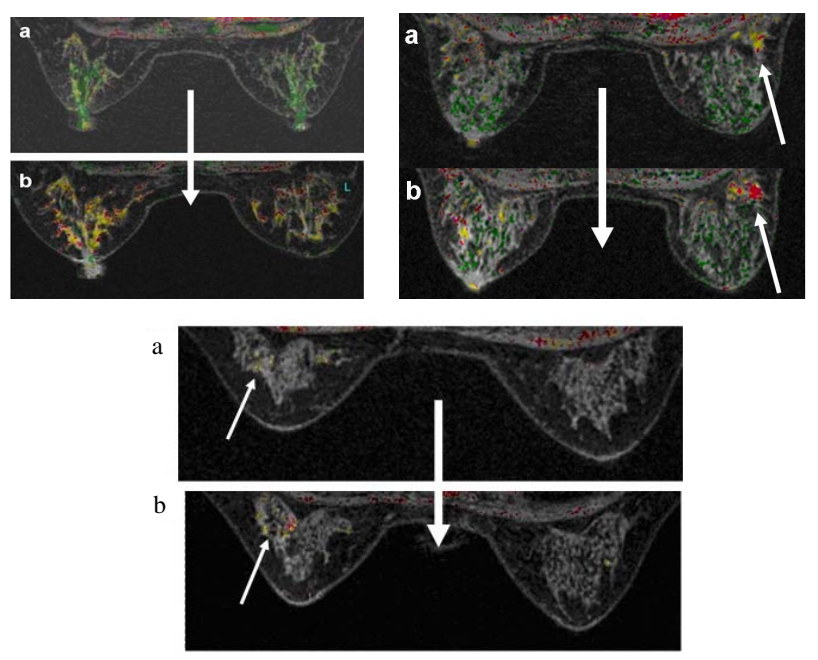

Figure 1. (a-c, left to right) Breast MRI of glandular tissue before (upper figures); and after (lower figures) intake of estrogen or phytoestrogen supplements of three cases. that may occur after the patient ceases to take estrogen or phytoestrogen supplements. One patient (Figure 3) initially showed a focal proliferative lesion in the left breast, with a benign teratoma found in her left ovary at the same time. She received a left ovariectomy, and the resulting decrease in the effect of endogenous estrogen was visible in her follow-up breast MRI, with significant improvement in the left breast being noted.

\section{DISCUSSION}

Prior research has demonstrated that in women, low serum estrogen concentrations are associated with a low incidence of breast cancer; in contrast, higher levels of serum estrogen are associated with higher incidence of breast cancer $[9,10]$.

Among Taiwanese women, exposure to estrogen has increased in the form of westernized diets rich in estrogen, an earlier pubertal age than in prior eras, delayed menopause, and obesity after menopause. Estrogen plays a role in promoting glandular cell proliferation, which
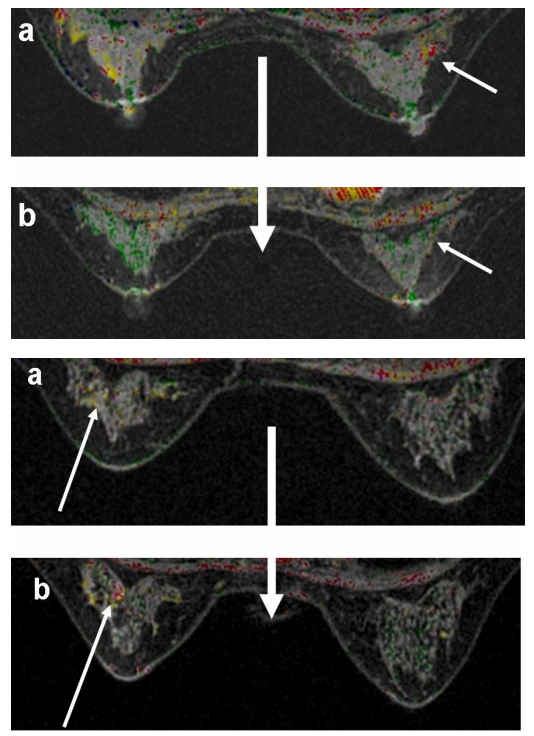

Figure 2. (a-b, left to right): Breast MRI of glandular tissue during intake of estrogen or phytoestrogen supplements (upper figures); and after ceasing intake (lower figures) of two cases.

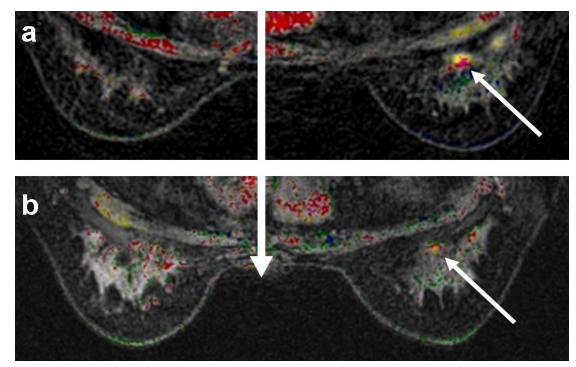

Figure 3. Breast MRI of glandular tissue, showing focal proliferative enhancement in the left breast (upper figure); after left ovariectomy, significant improvement was noted (lower figure). 
increases the risk of breast cancer [10-12].

Some studies have reported that dietary and nutritional factors account for approximately half of all breast cancer cases $[11,12]$. In the past, Asian diets may have facilitated a lower breast cancer rate, but in Taiwan this advantage has disappeared because of the adoption of westernized diets, over-nutrition, and overuse of herbal or traditional Chinese herbal medicines or other supplements that mimic estrogen components or phytoestrogen [11,12].

Women with glandular proliferative disease of the breast are at almost twice the risk for malignancy compared with the general population (including women with non-proliferative lesions). The breast cancer risk for women with atypical hyperplasia (proliferative lesions) is 5 times greater than that of women with non-proliferative lesions. Women with atypical ductal hyperplasia and a family history of breast cancer are at 11 times the risk compared with women who have a non-proliferative lesion and without a positive family history [13].

Many of our patients have told us that they believed that products from health food stores should be safe to use because they are "natural" or extracted from plants. However, hormone imbalances may be provoked by plants, health foods or herbs that mimic estrogen. Our clinical observations suggest that many young women begin to complain of sore or tender breasts and swelling before menstruation after they have started using dietary supplements that contain estrogen components or phytoestrogen.

Breast glandular cells contain cell membrane receptors for estrogen and other receptors for progesterone. Approximately 3 quarters of all breast cancers are estrogenreceptor positive (ER-positive), which means estrogen causes these tumors to grow. However, during malignant transformation, breast cancer cells do not distinguish between estrogen and phytoestrogen ingested by the patient [14-16].

Synthetic chemical estrogen (such as xenoestrogen) may take months to metabolize and pass out of the patient's body. The use of chemical estrogens has been found to correlate strongly with an increased incidence of breast cancer. In contrast, most food grade estrogen supplements or phytoestrogens are metabolized and pass out of the body in several days to weeks. Thus, short-term use of phytoestrogens may be poorly correlated with breast cancer incidence, but long-term or over-use of the same products may not be safe. Although extracted from plant tissue, phytoestrogen supplements intended to reduce menopausal symptoms may not provide protection from breast cancer [17-19].

Studies conducted with human breast cancer cell lines indicate that genistein both inhibits and stimulates proliferation of these cells. A mitogenic effect of genistein was observed at low doses and an antiproliferative effect at higher doses. The growth of murine breast cancer cell lines with estrogen-positive receptor (MCF-7 cells) was first stimulated and then inhibited by genistein, in a dosedependent manner [14-16]. One study found that genistein exerted different effects on the breast depending on the timing of exposure. In addition, phytoestro-gen-genistein may interfere with the inhibitive effects of tamoxifen on breast cancer cell growth [20]. A potential cancerpromoting effect of genistein should not be omitted [21]. In Asian regions, including Mainland China, Taiwan, Hong Kong and Singapore, many women seek natural supplements or traditional Chinese herbal support for the relief of menopause symptoms [17]. However, phytoestrogen extracted from natural sources or traditional Chinese herbs may stimulate the proliferation of breast cancer cell line (MCF-7) [21]. Several studies have analyzed the effects of various herbal supplements and medications. Cell cycle analysis has revealed that the stimulation of proliferation may be associated with a marked increase in the replication of MCF-7 cells, with the effects being similar to that of human estradiol [22-25].

\section{CONCLUSION}

This retrospective study of breast MRI images has demonstrated the similarity between synthetic HRT and supplements containing estrogen components, including Chinese herbs or phytoestrogen. It is important for nutritionists, naturopathic physicians, and Chinese herbal doctors to be aware of the problem of overuse of supplements containing estrogen components or phytoestrogen, or those mimic estrogen effects. Such supplements can increase the proliferation of breast glandular tissue and may increase the risk of breast cancer. Patients with a family history or personal history of breast cancer should be advised to strictly avoid any type of estrogen or phytoestrogen intake, unless it is essential.

\section{ACKNOWLEDGEMENTS}

The authors gratefully acknowledge the technical support to this study by Chen Yi-Fan and Chuang Chun-Yu.

\section{REFERENCES}

[1] Katalinic, A. and Rawal, R. (2008) Epidemiology decline in breast cancer incidence after decrease in utilisation of hormone replacement therapy. Breast Cancer Research Treatment, 107, 427-430. doi:10.1007/s10549-007-9566-Z

[2] Leung, T.K., Huang, P.J., Chen, C.S., Lin, Y.H., Wu, C.H. and Lee, C.M. (2010) Is breast MRI screening more effective than digital mammography in Asian women? Journal of Experimental \& Clinical Medicine, 2, 245-250. doi:10.1016/S1878-3317(10)60038-1

[3] Erguvan-Dogan, B., Whitman, G.J., Kushwaha, A.C., 
Phelps, M.J. and Dempsey, P.J. (2006) BI-RADS-MRI: A primer. American Journal of Roentgenology, 187, W152W160. doi:10.2214/AJR.05.0572

[4] Stephens, T.W. (1998) Illustrated breast imaging reporting and data system (BI-RADS). 3rd Edition, American College of Radiology, Reston.

[5] Liberman, L. and Menell, J.H. (2002) Breast imaging reporting and data system (BI-RADS). Radiologic Clinics of North America, 40, 409-430. doi:10.1016/S0033-8389(01)00017-3

[6] Leung, T.K., Chu, J.S., Huang, P.J., Lee, C.M., Lin, Y.H., Chen, C.S., Tai, C.J. and Wu, C.H. (2010) Breast MRI for monitoring images of an "adenomyoepithelioma with malignant features”, before, during, and after chemotherapy. Breast Journal, 16, 652-653. doi:10.1111/j.1524-4741.2010.01009.x

[7] Leung, T.K., Huang, P.J., Lee, C.M., Chen, C.S., Wu, C.H. and Chao, J.S. (2010) Can breast magnetic resonance imaging demonstrate characteristic findings of preoperative ductal carcinoma in situ in Taiwanese women? Asian Journal of Surgery, 33, 143-149. doi:10.1016/S1015-9584(10)60024-3

[8] Leung, T.K., Huang, P.J., Lee, C.M. and Chen, C.S. (2012) Silicone breast implant with intracapsular rupture coexisting with locally advanced carcinoma. Breast Journal, 18, 76-77. doi:10.1111/j.1524-4741.2011.01187.x

[9] Key, T.J., Chen, J., Wang, D.Y., Pike, M.C. and Boreham, J. (1990) Sex hormones in women in rural China and in Britain. British Journal of Cancer, 62, 631-636. doi:10.1038/bjc.1990.344

[10] Clemons, M. and Goss, P. (2001) Estrogen and the risk of breast cancer. New England Journal of Medicine, 344, 276-285. doi:10.1056/NEJM200101253440407

[11] Tsai, A.C., Liou, J.C., Chang, M.C. and Chuang, Y.L. (2007) Influence of diet and physical activity on aging-associated body fatness and anthropometric changes in older Taiwanese. Nutrition Research, 27, 245-251. doi:10.1016/j.nutres.2007.03.002

[12] Hsieh, S.C., Lai, J.N., Lee, C.F., Hu, F.C., Tseng, W.L. and Wang, J.D. (2008) The prescribing of Chinese herbal products in Taiwan: A cross-sectional analysis of the national health insurance reimbursement database. Pharmacoepidemiological Drug Safety, 17, 609-619. doi:10.1002/pds.1611

[13] Dupont, W.D. and Page, D.L. (1985) Risk factors for breast cancer in women with proliferative breast disease. New England Journal of Medicine, 312, 146-151.

\section{doi:10.1056/NEJM198501173120303}

[14] Hsieh, C.Y., Santell, R.C., Haslam, S.Z. and Helferich, W.G. (1998) Estrogenic effects of genistein on the growth of estrogen receptor-positive human breast cancer (MCF7) cells in vitro and in vivo. Cancer Research, 58, 38333838 .

[15] Zava, D.T. and Duwe, G. (1997) Estrogenic and antiproliferative properties of genistein and other flavonoids in human breast cancer cells in vitro. Nutrition Cancer, 27, 31-40. doi:10.1080/01635589709514498

[16] Wang, T.T., Sathyamoorthy, N. and Phang, J.M. (1996) Molecular effects of genistein on estrogen receptor mediated pathways. Carcinogenesis, 17, 271-275. doi:10.1093/carcin/17.2.271

[17] Yager, J.D. and Davidson, N.E. (2006) Estrogen carcinogenesis in breast cancer. New England Journal of Medicine, 354, 270-282. doi:10.1056/NEJMra050776

[18] Peeters, P.H.M., Keinan-Boker, L., van der Schouw, Y.T. and Grobbee, D.E. (2003) Phytoestrogens and breast cancer risk: Review of the epidemiological evidence. Breast Cancer Research Treatment, 77, 171-183. doi:10.1023/A:1021381101632

[19] Keinan-Boker, L., van der Schouw, Y.T., Grobbee, D.E. and Peeters, P.H.M. (2004) Dietary phytoestrogens and breast cancer risk. American Journal of Clinical Nutrition, 79, 282-288.

[20] Duffy, C., Perez, K. and Partridge, A. (2007) Implications of phytoestrogen intake for breast cancer. $\mathrm{Ca}$-A Cancer Journal for Clinicians, 57, 260-277. doi:10.3322/CA.57.5.260

[21] Bouker, K.B. and Hilakivi-Clarke, L. (2000) Genistein: Does it prevent or promote breast cancer? Environmental Health Perspectives, 108.

[22] Mayo, J.L. (1997) A Natural approach to menopause. Clinical Nutrition Insights, 5, 1-8.

[23] Huang, K.C. (1993) The pharmacology of Chinese herbs. CRC Press, Boca Raton.

[24] Yapa, S.P., Shena, P., Lia, J., Leeb, L.S. and Yonga, E.L. (2007) Molecular and pharmacodynamic properties of estrogenic extracts from the traditional Chinese medicinal herb, Epimedium. Journal of Ethnopharmacology, 113, 218-224. doi:10.1016/j.jep.2007.05.029

[25] Wang, Z.Q. and Lou, Y.J. (2004) Proliferation-stimulating effects of icaritin and desmethylicaritin in MCF-7 cells. European Journal of Pharmacology, 504, 147-153. doi:10.1016/j.ejphar.2004.10.002 